\title{
Insights into the COVID-19 pandemic from a rare neurodegenerative disease
}

Stephen L. Sturley ${ }^{1}$, Zsuzsa Márka ${ }^{2}$, Szabolcs Márka $^{2}$, Natalie Hammond ${ }^{3}$, Tamayanthi Rajakumar $^{3}$ and Andrew B. Munkacsi ${ }^{3}$

${ }^{1}$ Department of Biology, Barnard College, 3009 Broadway, New York, NY 10027; ${ }^{2}$ Department of Physics, Columbia University, 538 West 120th Street, 1009 Pupin, MC 5229, Box 29, New York, NY, 10027; and ${ }^{3}$ School of Biological Sciences and Centre for Biodiscovery, Victoria University of Wellington, Wellington, New Zealand 6012.

Keywords: Coronavirus, COVID-19, SARS-CoV, EBOV, pandemic, drug repurposing, cholesterol, neurodegenerative disease, lysosomal storage disease 


\section{Summary}

The current justified furor regarding epidemic preparedness has emphasized an unmet need for therapeutic strategies that lead to rapid implementation. The prompt identification and sequencing of the COVID-19 genome has prompted herculean efforts to rapidly design and produce vaccines and other antiviral interventions. Nevertheless, the generalized availability of such therapeutics will be markedly out of phase with the first wave of this disease. Strategic drug repurposing combined with rapid testing of molecular targets could provide at least a pause in disease progression and aid mitigation. With respect to the current pandemic, COVID-19, in common with other enveloped viruses (e.g. HIV, Ebola, SARS, and MERS) encounter the endosomal/lysosomal host compartment as a critical step for infection and maturation. A surprisingly key interaction requires engagement of the Niemann-Pick type C1 (NPC1) pathway. This observation prompts the utilization of NPC1 inhibitors as antiviral agents. Fortunately, there are such molecules, in many cases available as generics that could be rapidly deployed in the upcoming months. Herein we expand upon this strategy and its scientific underpinning wherein given the biological conservation of SARS-CoV-1 and SARS-CoV-2, and the role of the NPC1-dependent late endosome/lysosome lipid pathway in coronavirus infections, we suggest that the known mechanistic information on NPC1 could be utilized within the context of COVID-19 and associated candidate therapeutics. 
While a world-wide efficacious vaccination program for emerging diseases such as coronavirus disease 2019 (COVID-19), would likely eradicate the disease, implementation will be dauntingly distant as well as challenged by newly evolving mutations in the virus. Similarly, novel anti-infective drug design, even when optimized for intervention against well researched molecular pathways is likely to face a prolonged path to approval. Drug repurposing, coupled with strategic assessments of molecular targets, is one research approach by which both containment and mitigation of this current global outbreak could be advanced; this tactic suggested several existing, clinically approved drugs for treating COVID-19 ${ }^{1}$ that are currently being prescribed "off-label" or in clinical trial. Given the lipid-regulating activity of some of these therapeutics (e.g., Chloroquine) and the lipid-localization of the host cell receptor for SARS-CoV-2 (angiotensinconverting enzyme, ACE2), it is surprising that to date, the role of lipids has not been prioritized. Here we provide such insight that could be investigated as candidate treatments for the current COVID-19 pandemic caused by coronavirus SARS-CoV-2.

Our insight into the life cycles of the majority of infectious agents is remarkably sophisticated, often informed by knowledge about the specific organism or one closely related. Of timely relevance to the current pandemic, it is known that enveloped viruses (HIV, Ebola (EBOV), MERS-CoV, SARS-CoV-1 and SARS-CoV-2) disassemble their proteolipid coat at the plasma membrane (PM) or in the lysosome of infected cells, thereby liberating their genetic information for dissemination and formation of new virions (Figure 1). This cycle can be interrupted by its dependency on host factors that mediate endocytosis and recycling, in which lipid homeostasis plays a key role. Numerous studies suggest that ablation of the Niemann-Pick type C1 (NPC1) pathway confers resistance to a multiplicity of viral infections and potentially amelioration of symptoms. The human NPC1 protein comprises 13 transmembrane domains that traverse the limiting membrane of the late endosome/lysosome (LE/L) and plays a vital role in intracellular lipid transport by facilitating the mobilization of cholesterol and sphingolipids. Loss of function of NPC1 causes the fatal, pediatric Niemann-Pick type C (NP-C) disease - whereby lipid accumulation in the LE/L impairs organelle function leading to a cascade of visceral and neurodegenerative symptoms including dementia. In a startling example of how research into rare diseases can extend into a larger arena, a series of investigations identified a commonality between NP-C disease and the life cycle of numerous viruses such as SARS-CoV, MERS-CoV, EBOV and HIV. Interestingly, the life cycle of these viruses is impeded by loss of NPC1. Based on resistance 
of $\mathrm{Npcl}^{+/}$mice to EBOV 2, persons with half-normal levels of the NPC1 protein (i.e., heterozygotes) are anticipated to be resistant to the viral disease - a concept known as heterozygote advantage.

NP-C disease is surprisingly well studied given its rarity (only 500 cases worldwide); for decades research scientists have used drugs as research tools to model the disease, some of which are clinically approved in the treatment of unrelated disorders. Impressively, a number of these NPC1 inhibitors reduce the infectivity of numerous viruses by hindering viral release into the cytosol and preventing viral replication. The host cell receptor (i.e., ACE2 in SARS-CoV-1 and SARS-CoV-2) and proteases (i.e., TMPRSS2 in SARS-CoV-1 and SARS-CoV-2) that facilitate SARS-CoV-2 entry into the host cell (early viral entry) are localized in cholesterol- and sphingolipid-rich microdomains ${ }^{3-5}$. Inhibiting NPC1 depletes these microdomains, thus possibly disturbing the dispersion of the host cell receptors and proteases in the PM, and therefore reducing binding/priming of viral S protein for entry (Figure 1). Alternatively, the S protein in SARS-CoV2 is primed by cathepsin $\mathrm{B} / \mathrm{L}$ in the $\mathrm{LE} / \mathrm{L}$ for viral entry through the endosomal pathway (late viral entry) ${ }^{4}$. In this case, NPC1 inhibition may hinder the release of SARS-CoV-2 from the LE/L (Figure 1), whether this be directly (as in EBOV) or as a result of cholesterol accumulation causing impaired LE/L function is not yet known. Indeed, blocking both the entry from the cell surface and through the endosomal pathway, exhibited efficient inhibition of SARS-CoV-1 infection ${ }^{6}$. Therefore, inhibiting NPC1 enables a dual blockade of viral entry into host cells and thus represents a means to inhibit SARS-CoV-2 infectivity.

Numerous, readily available, NPC1 inhibitors are effective in limiting viral infections. The NPC1 inhibitor U18666A (a cationic amphiphile) dramatically reduced infectivity of SARS-CoV1, EBOV, Influenza A, Chikungunya, Hepatitis C, Zika, Dengue and HIV 7-10. Specifically, U18666A strongly inhibited S protein-driven entry of SARS-CoV-1 into host cells ${ }^{11}$. Imipramine (an amphiphilic drug clinically approved to treat depression) or Itraconazole (a clinically approved antifungal drug) effectively blocked the activity of NPC1 and reduced infectivity of coronavirus and filoviruses ${ }^{2,7,12,13}$. Similarly, Cepharanthine (an approved anti-inflammatory and anti-cancer drug) exhibited complete inhibition of the pangolin coronavirus model for COVID-19 ${ }^{14}$, perhaps by binding and inhibition of NPC1 ${ }^{15}$. An alternate approach is to use therapeutics that alter cholesterol homeostasis and mimic NP-C disease. Interestingly, Chloroquine (a cationic amphiphile like U18666A), a top contender of all approved compounds to treat COVID-19, 
mimics NP-C disease via inhibiting cholesterol esterification and autophagy - this clearly implicates NPC1 in a potential treatment for inhibiting SARS-CoV-2 ${ }^{1}$. Additionally, other cationic amphiphiles Chlorpromazine (approved to treat schizophrenia and depression) and Amiodarone (approved to treat cardiac arrhythmia) could be tested as candidate therapies for treating COVID-19; both compounds mimic NP-C disease, and inhibited SARS-CoV-1 and MERS-CoV ${ }^{16,17 .}$

In summary, given the biological conservation of SARS-CoV-1 and SARS-CoV-2, and the role of the NPC1-dependent LE/L lipid pathway in coronavirus infections, we suggest that the known mechanistic information on NPC1 could be utilized within the context of COVID-19 and associated candidate therapeutics. Rapid and efficacious drug repurposing of any of the established globally prescribed therapeutics that are also NPC1 inhibitors or NP-C disease mimics, might contribute to global relief from the health consequences of this pandemic, while the march toward an effective vaccine proceeds. 


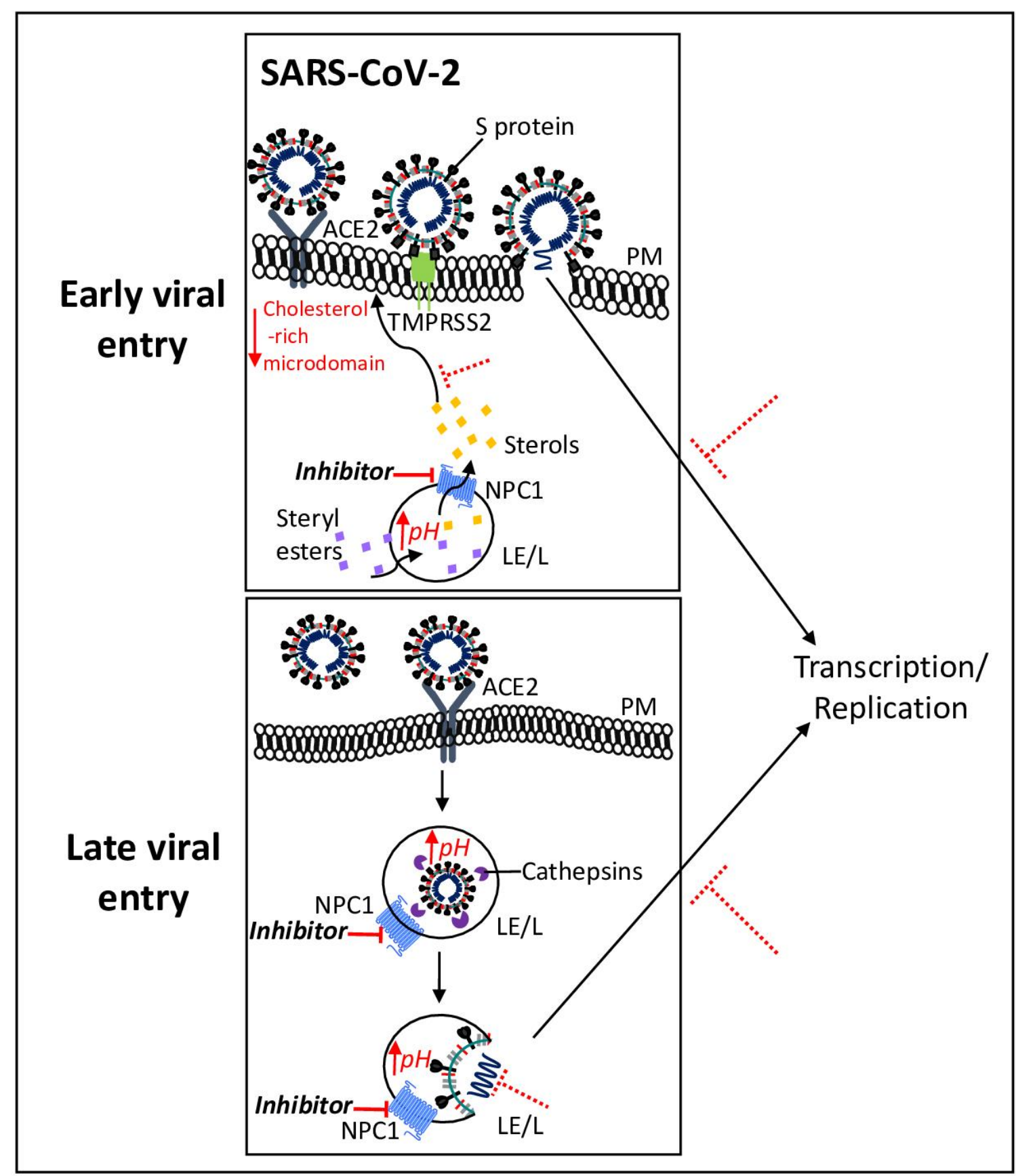

Figure 1. Proposed dual inhibition of SARS-CoV-2 entry/replication by inhibiting NPC1. Early viral entry of SARS-CoV-2 is mediated by TMPRSS2 (green) which primes the S protein upon binding to ACE2 (grey). For late viral entry, cathepsins (purple) prime the $\mathrm{S}$ protein prior to release from the late endosome/lysosome (LE/L) for transcription/replication. Normally, NPC1 functions in cholesterol egress from the LE/L to plasma membrane (PM). Inhibiting NPC1 (solid red line) depletes levels of cholesterol at the PM, affecting the binding and priming (early viral entry) (dashed red line) and or the LE/L (late viral entry). Additionally, inhibiting NPC1 leads to cholesterol accumulation that impairs LE/L function (e.g., increasing $\mathrm{pH}$ ) - both of which impede viral transcription/replication (dashed red line). 


\section{Contributors}

SLS initiated this Comment and all authors contributed equally to the final manuscript.

\section{Funding}

We thank the Ara Parseghian Medical Research Foundation (to SLS and ABM), Dana's Angels Research Trust (to SLS), National Institutes of Health (DK54320 to SLS), Wellington Medical Research Foundation (to ABM), and Research For Life (to ABM). ABM was supported as a Peter Pentchev Research Fellow of the National Niemann-Pick Disease Foundation, a Senior Fellow in Biomedical Sciences of the Charles Revson Foundation, and an NIH Postdoctoral Fellow in Arteriosclerosis (Grant T32 HL07343).

\section{Declaration of interests}

We declare no competing interests. SLS is a member of the scientific advisory board of the National Niemann-Pick Disease Foundation. 


\section{References}

1. Liu C, Zhou Q, Li Y, et al. Research and development on therapeutic agents and vaccines for COVID-19 and related human coronavirus diseases. ACS Cent Sci 2020; In Press.

2. Herbert AS, Davidson C, Kuehne AI, et al. Niemann-Pick C1 is essential for Ebolavirus replication and pathogenesis in vivo. mBio 2015; 6: e00565-15.

3. Glende J, Schwegmann-Wessels C, Al-Falah M, et al. Importance of cholesterol-rich membrane microdomains in the interaction of the $\mathrm{S}$ protein of SARS-coronavirus with the cellular receptor angiotensin-converting enzyme 2. Virology 2008; 381: 215-21.

4. Hoffmann M, Kleine-Weber H, Schroeder S, et al. SARS-CoV-2 cell entry depends on ACE2 and TMPRSS2 and is blocked by a clinically proven protease inhibitor. Cell 2020; In Press. 5. Walls AC, Park YJ, Tortorici MA, Wall A, McGuire AT, Veesler D. Structure, function, and antigenicity of the SARS-CoV-2 spike glycoprotein. Cell 2020; In Press.

6. Kawase M, Shirato K, van der Hoek L, Taguchi F, Matsuyama S. Simultaneous treatment of human bronchial epithelial cells with serine and cysteine protease inhibitors prevents severe acute respiratory syndrome coronavirus entry. J Virol 2012; 86: 6537-45.

7. Wichit S, Hamel R, Bernard E, et al. Imipramine inhibits Chikungunya virus replication in human skin fibroblasts through interference with intracellular cholesterol trafficking. Sci Rep 2017; 7: 3145.

8. Lu F, Liang Q, Abi-Mosleh L, et al. Identification of NPC1 as the target of U18666A, an inhibitor of lysosomal cholesterol export and Ebola infection. eLife 2015; 4: e12177.

9. Tang Y, Leao IC, Coleman EM, Broughton RS, Hildreth JE. Deficiency of Niemann-Pick type C-1 protein impairs release of human immunodeficiency virus type 1 and results in Gag accumulation in late endosomal/lysosomal compartments. J Virol 2009; 83: 7982-95.

10. Eckert N, Wrensch F, Gartner S, et al. Influenza A virus encoding secreted Gaussia luciferase as useful tool to analyze viral replication and its inhibition by antiviral compounds and cellular proteins. PLoS ONE 2014; 9: e97695.

11. Wrensch F, Winkler M, Pohlmann S. IFITM proteins inhibit entry driven by the MERScoronavirus spike protein: evidence for cholesterol-independent mechanisms. Viruses 2014; 6: 3683-98.

12. Takano T, Akiyama M, Doki T, Hohdatsu T. Antiviral activity of itraconazole against type I feline coronavirus infection. Vet Res 2019; 50: 5.

13. Trinh MN, Lu F, Li X, et al. Triazoles inhibit cholesterol export from lysosomes by binding to NPC1. Proc Natl Acad Sci USA 2017; 114: 89-94.

14. Fan HH, Wang LQ, Liu WL, et al. Repurposing of clinically approved drugs for treatment of coronavirus disease 2019 in a 2019-novel coronavirus (2019-nCoV) related coronavirus model. Chin Med J 2020; In Press.

15. Lyu J, Yang EJ, Head SA, et al. Pharmacological blockade of cholesterol trafficking by cepharanthine in endothelial cells suppresses angiogenesis and tumor growth. Cancer Lett 2017; 409: 91-103.

16. Stadler K, Ha HR, Ciminale V, et al. Amiodarone alters late endosomes and inhibits SARS coronavirus infection at a post-endosomal level. Am J Respir Cell Mol Biol 2008; 39: 142-9.

17. de Wilde AH, Jochmans D, Posthuma CC, et al. Screening of an FDA-approved compound library identifies four small-molecule inhibitors of Middle East respiratory syndrome coronavirus replication in cell culture. Antimicrob Agents Chemother 2014; 58: 4875-84. 